\title{
Universidad inclusiva: percepciones de los responsables de los servicios de apoyo a las personas con discapacidad ${ }^{1}$ Inclusive University: Perceptions of Disability Support Services
}

\author{
Odet Moliner García (1) molqar@edu.uji.es \\ María Angélica Yazzo Zambrano (2) mayazzoz@libertadores.edu.co \\ Daniel Niclot (3) daniel.niclot@univ-reims.fr \\ Thierry Philippot (3) thierry.philippot@univ-reims.fr \\ (1) Universitat Jaume I \\ (2) Fundación Universitaria Los Libertadores \\ (3) Université de Reims Champagne-Ardenne \\ (Recibido: 10 de mayo de 2017; Aceptado para su publicación: 4 de agosto de 2017)
}

Cómo citar: Moliner, O., Yazzo, M. A., Niclot, D. y Philippot, T. (2019). Universidad inclusiva: percepciones de los responsables de los servicios de apoyo a las personas con discapacidad. Revista Electrónica de Investigación Educativa, 21, e20, 1-10. doi:10.24320/redie.2019.21.e20.1972

\section{Resumen}

Este artículo presenta los resultados de una investigación efectuada en tres universidades de España, Colombia y Francia sobre las percepciones del personal de los servicios de apoyo a las personas con discapacidad en la universidad. Se trata de un estudio descriptivo en el que, mediante entrevistas individuales, los directores académicos y los técnicos de los servicios expresan cómo entienden sus prácticas en relación con el apoyo que proporcionan al estudiantado y al profesorado desde el análisis de tres dimensiones: a) cultura, b) política y c) prácticas inclusivas en la universidad. El análisis de los resultados pone de manifiesto las barreras y fortalezas encontrados en su trabajo. Se concluye con una serie de retos que orientan futuras acciones en cada contexto.

Palabras clave: Servicios de apoyo, discapacidad, educación superior.

\section{Abstract}

This article presents the results of a study carried out in three universities in Spain, Colombia, and France on the perceptions of disability support staff. In this descriptive study, individual interviews were conducted in which academic directors and disability support workers described how they perceived their work with respect to support provided to students and teachers. Three dimensions are discussed: a) culture; b) policies; and c) inclusive practices in the university. The analysis of the results highlights the obstacles and strengths identified within their work. To conclude, the article lists challenges that may guide future action in each context.

Keywords: Support services, disability, higher education.

\footnotetext{
1 Investigación financiada por la Oficina de Cooperación al Desarrollo de la Universitat Jaume I. Convocatoria de Proyectos del Observatorio Permanente de la inmigración OPI-UJ 2016.
} 


\section{Introducción}

En el cumplimiento del derecho a la educación inclusiva, el artículo 24 de la Convención Internacional de los Derechos de las Personas con Discapacidad de 2006 establece incorporar en el sistema educativo el enfoque inclusivo en todos los niveles de enseñanza. Por tanto, las instituciones de educación superior deben asegurar el acceso, la permanencia y la graduación oportuna con ajustes razonables, la formación efectiva, el objetivo de la plena inclusión, participación plena y en igualdad de condiciones en la educación y aprendizaje durante toda la vida. A partir de entonces cada país implementa un conjunto de políticas asumiendo la ratificación del artículo.

En el caso de España, la reforma del sistema a raíz del Espacio Europeo de Educación Superior (EEEs), planteó como uno de los indicadores de calidad el acceso igualitario a la enseñanza superior de las personas con discapacidad. Desde la Ley Orgánica de Universidades 4/2007, en concreto desde la disposición adicional vigésimo cuarta, se describe cómo debe ser la inclusión de las personas con discapacidad en las universidades. Además, el Real Decreto 1892/2008, del 14 de noviembre, por el que se regulan las condiciones para el acceso a las enseñanzas universitarias oficiales, mantiene esta filosofía en sus textos haciendo que la atención a la diversidad en la universidad española esté claramente regulada para su puesta en funcionamiento.

En Francia, la ley del 11 de febrero de 2005 para la igualdad de derechos y oportunidades, participación y ciudadanía de las personas con discapacidad, organizó la política de la escolarización de los alumnos y los estudiantes con discapacidad. El artículo 20 de esta ley se refiere directamente a las universidades y las obliga a garantizar a los estudiantes con discapacidad o con un problema de salud o minusvalía, los mismos derechos que al resto de los estudiantes. Posteriormente se firmó una primera Carta Universidad-Discapacidad en 2007 entre las universidades, el Ministerio de Educación Superior e Investigación, el Ministerio de Trabajo, Relaciones Sociales y solidaridad. Cinco años después, en mayo de 2012, se firmó un estatuto que establece una política de inclusión de la discapacidad y garantizar la igualdad de oportunidades de los estudiantes como personas independientemente de su condición.

En Colombia, el estado ratifica el derecho de las personas con discapacidad con la Ley estatuaria 1618 de 2013, que en su artículo 11 proyecta el acceso a la educación superior, la formación profesional, la educación para adultos, la educación para el trabajo y el aprendizaje durante la vida. Por esta ley el Ministerio de Educación emite los lineamientos de política superior inclusiva en el año 2013 para todas las instituciones educativas a nivel superior a través de un enfoque diferencial.

Así pues, la inclusión de estudiantes con discapacidad en las universidades es un hecho social relevante que se ha constituido en objeto de investigación en el ámbito internacional. El trabajo de Douchet, Aubree y Mabaka (2013) ofrece un interesante cuadro comparativo de las políticas de inclusión en las universidades de todo el mundo, teniendo en cuenta los resultados en Europa, América, Asia y algunos países africanos. Sin embargo, numerosos estudios (Leyser, Greenberger, Sharoni y Vogel, 2011; Moswela y Mukhopadhyay, 2011; Prowse, 2009; Riddell y Weedon, 2014; Shevlin, Kenny y Mcneela, 2004) muestran que las universidades, en ocasiones, no están suficientemente preparadas para incluir a todos los estudiantes desde un enfoque inclusivo.

Con respecto a la situación específica de Francia, parece ser un tema ausente de las preocupaciones de los investigadores, con alguna salvedad de algún trabajo empírico sobre el tema (Grosstephan y Philippot, 2015), que conecta los dispositivos desarrollados en las universidades y las prácticas de los actores en un estudio de caso. También un informe institucional de la Inspección General de la Educación Nacional e Investigación, de 2006, ofrece una evaluación crítica de los resultados de las políticas implementadas.

En el contexto español se han realizado algunos estudios previos (Castellana y Sala, 2005; Moriña, CortesVega y Molina, 2015) concluyen que las aulas no disponen de los recursos necesarios para favorecer la inclusión, el profesorado no imparte las clases utilizando las metodologías adecuadas para que puedan participar plenamente en ellas dificultando a los estudiantes con discapacidad la participación, el progreso y el éxito en la universidad.

En Colombia se aprecia un incremento del interés sobre este tema de investigación a partir de los Lineamientos en Política de Educación Superior Inclusiva del Ministerio de Educación Nacional de 2013 y proliferan algunos trabajos, como el de Yarza, Rojas y López (2014) y el proyecto denominado "La 
inclusión educativa de personas con discapacidad: políticas y prácticas en la educación superior. Estudio comparativo entre la Universidad Católica de Córdoba-Argentina y la Fundación Universitaria Los Libertadores-Colombia" cuyo objetivo es identificar el impacto de las políticas y prácticas educativas inclusivas establecidas en los procesos de educación superior para el acceso, permanencia y egreso de las poblaciones con discapacidad (Rodríguez, 2015).

Por eso es fundamental el rol de los servicios de apoyo en la promoción de universidades inclusivas. Estos servicios, programas y unidades de atención se caracterizan por su heterogeneidad y el desempeño de sus funciones varía considerablemente de unos contextos a otros, incluso dentro de un mismo país (Alonso y Díez, 2008). Entre sus competencias se incluye la de actuar como mediadores entre estudiantes y profesorado, orientar y dar soporte a estudiantes y profesores, sensibilizar y formar al profesorado y velar para la realización de las adaptaciones y ajustes necesarios para la permanencia y progreso de los estudiantes. Así pues, desempeñan su rol en tres dominios o dimensiones que son centrales para el desarrollo del enfoque inclusivo (Booth y Ainscow, 2002):

a) Cultura inclusiva referida a los valores inclusivos compartidos por la institución. Los principios que se derivan de esta cultura universitaria son los que guían las decisiones que se concretan en las políticas.

b) Política inclusiva referida a la organización institucional: normativas, servicios y recursos disponibles en la institución que garantizan el acceso, la participación y el aprendizaje de todos los estudiantes.

c) Práctica inclusiva más enfocada a la acción docente. Incluye un planteamiento transversal de los principios del DUA (Diseño Universal del Aprendizaje).

Los responsables de los servicios de apoyo trabajan estrechamente con los responsables políticos, los estudiantes y el profesorado, y articulan los recursos y condiciones para que cada institución desarrolle una cultura más o menos inclusiva. Sin embargo, como nos recuerdan algunos autores (Borland y James, 1999; Moriña et al., 2015) el ambiente universitario puede ser en algunos casos discapacitador. En este sentido, la presente investigación pretende contribuir al estudio de la articulación de las culturas, políticas y prácticas inclusivas en la universidad, poniendo el foco en las percepciones de los profesionales de los servicios de apoyo.

\section{Método}

Presentamos un estudio empírico sobre las percepciones de los responsables de los servicios de apoyo a las personas con discapacidad en la universidad sobre el desarrollo de la educación inclusiva en la educación superior.

En la Universitat Jaume I la Unidad de Soporte Educativo (USE) funciona desde 1992 como un servicio que pretende dar una respuesta personalizada y eficiente a las necesidades del alumnado y del profesorado. Se estructura en cuatro ámbitos: información, orientación, formación del profesorado e innovación educativa y el Programa de Atención a la Diversidad (PAD). Este último se centra en el trabajo con las personas con necesidades educativas específicas y todo su entorno educativo universitario (profesorado, compañeros de estudios, entorno físico, entorno social, cultural y educativo). Se consideran las necesidades educativas específicas relacionadas con la discapacidad sensorial auditiva o visual, la movilidad reducida o la discapacidad motora, así como las enfermedades crónicas, problemáticas psíquicas o psicológicas y situaciones personales difíciles de incapacidad temporal (fracturas, operaciones, rehabilitación...) que repercuten directamente en el estudio o trabajo académico.

En la Fundación Universitaria Los Libertadores, en el año 2013 inició el Programa de Permanencia y Graduación Oportuna (PYGO) como estrategia para garantizar que todos los estudiantes puedan concluir su formación profesional con pertinencia. Por ello se estableció un sistema de alertas que identifican las condiciones de riesgo para la deserción a nivel económico, familiar, emocional y académico. Del 2014 al 2016, junto con la licenciatura de Educación Especial -y con el fin de fortalecer la atención de estudiantes con discapacidad y suprimir las barreras de aprendizaje-, se desarrolló la Política de educación superior inclusiva de la Fundación. Con ella surge la necesidad de generar la movilidad de pensamiento hacia un enfoque de inclusión como un proceso sistémico que posibilita la convivencia y el aprendizaje diverso, así 
como la articulación de recursos y programas.

En la Universidad de Reims Champagne-Ardenne (URCA), en 2008, se implementó la política de inclusión desde un enfoque de atención a la discapacidad, dirigida por un funcionario asignado por el rector y un coordinador de Servicio a la Discapacidad (SAD). También cuenta con un responsable de la calidad de vida laboral, referente de los trabajadores con discapacidad en la URCA, adscrito al Departamento de Recursos Humanos. La política está dirigida tanto a estudiantes como al personal con discapacidad de la universidad.

Esta investigación pretende analizar las percepciones de los responsables académicos y de los técnicos que gestionan los servicios de atención a la diversidad en las tres universidades implicadas, así como los retos percibidos que plantea la inclusión y que pueden ser mejorados. Se optó por un enfoque cualitativo, concretamente un estudio descriptivo realizado a partir de los datos obtenidos en entrevistas, las cuales se realizaron individualmente, previa firma del consentimiento informado.

El instrumento utilizado fue un guión de entrevista semiestructurada que constaba de tres apartados: a) datos contextuales, b) datos del informante y c) 35 preguntas agrupadas por indicadores sobre tres temas fundamentales relacionados con el concepto de inclusión y los valores de la institución (dimensión A), las políticas, estructura y organización (dimensión B), y las prácticas inclusivas (dimensión C). Por último, se lanzaba una pregunta abierta sobre los problemas o retos percibidos como más acuciantes. Las entrevistas tuvieron una duración aproximada de hora y media y fueron transcritas literalmente.

El muestreo de los informantes fue no probabilístico e intencional, al tratarse de expertos seleccionados como fuentes de información relevantes. Los participantes fueron 13 profesionales, con una experiencia muy variable en función de la posición que ocupaban en el servicio.

Tabla I. Descripción de la muestra

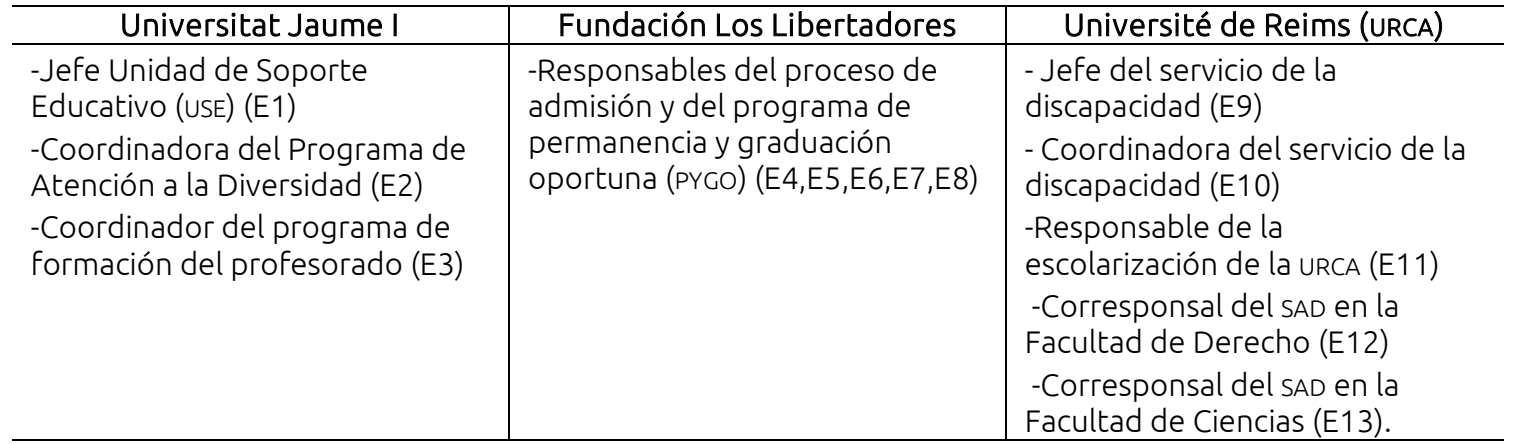

Los datos se analizaron a partir de la reducción de las transcripciones literales de las entrevistas en unidades de contenido (Sabourin, 2009). Mediante un proceso de categorización se asignaron las unidades de contenido a cada una de las subcategorías que conformaban las tres dimensiones. Por tanto, el proceso de análisis de contenido ha tenido carácter deductivo en función de los indicadores consensuados para cada dimensión objeto de indagación.

\section{Resultados}

\subsection{Percepciones de los responsables de los servicios de apoyo en la Universitat Jaume I}

Sobre la dimensión A (Cultura), los informantes parten de una definición clara de la educación inclusiva como aquella que se centra en el estudiante: "Teniendo en cuenta las particularidades de cada uno de ellos de manera que cada uno pueda participar plenamente, tomando decisiones y estar en el espacio que corresponda..." (E2). También consideran que tiene como objetivo "dar la igualdad de oportunidades a todos los alumnos con los que cuenta la universidad (E3), y que además se plantea como "un punto de encuentro en un contexto educativo, en el que la atención a la diversidad ha de estar presente en todos los niveles, desde la organización del centro a la formación del profesorado y a la práctica educativa en general (E1).

Consideran, además, que la educación inclusiva sí que está contemplada "sobre el papel", es decir: "El 
modelo educativo de la uJ sí que contempla la posibilidad de atender al alumno con necesidades" (E3), y que se realiza sensibilización mediante cursos, conferencias, un programa de radio de la universidad y con las redes sociales.

En cuanto a la dimensión B (política), se muestran orgullosos de que esta universidad sea pionera en el estado español al introducir en sus estatutos (en 1992) el apoyo a las personas con discapacidad física, psíquica o sensorial: "Fuimos innovadores, porque fuimos la primera universidad que contemplaba todos los aspectos y situaciones" (E2).

Respecto de las condiciones de acceso y detección del alumnado pueden darse diversas situaciones: "a) que sea una persona con discapacidad y que por tanto, en el momento de matricularse, tiene matrícula gratuita. Gracias al sistema de gestión centralizada, automáticamente los estudiantes ya nos aparecen en nuestro registro y en la base de datos; b) un estudiante que no tiene una discapacidad y no tiene certificado, pero viene a contarnos que tiene una cierta situación determinada, una enfermedad crónica, un TDAH [Trastorno por Déficit de Atención con Hiperactividad] por ejemplo, una depresión, lo que sea... pues en ese momento lo incorporamos; y c) una detección en el aula, es decir, que el profesorado lo remita al servicio" (E2).

Prestan atención directa a unos 300 estudiantes y en algunos casos se aplican medidas de discriminación positiva: "Por ejemplo, en la biblioteca hay una cabina para ciegos donde pueden trabajar con programas específicos para poder leer documentos" (E1).

Destacan la cultura colaborativa y el trabajo con otras entidades, sobre todo con redes de servicio como el de la SAPDU (Servicios de Apoyo a las Personas con Discapacidad en la Universidad) y UNIDISVAL (Servicios de Universidad Discapacidad en Valencia). También existe gran colaboración con otros servicios internos de la universidad: "Trabajamos con el Servicio de Deportes, con Gestión Académica, en el tema de matrículas estamos ahí, en selectividad formamos parte de tribunales, con la Oficina de Prácticas hemos creado un grupo de trabajo... ahora con movilidad también, con el Servicio de Informática" (E2). También con la OCD (Oficina de Cooperación y Desarrollo) porque ellos tienen el voluntariado... en esto no tenemos problema, al contrario, necesitamos 3 y se presentan 20" (E1).

Sin embargo, sobre la coordinación, consideran que: "Tendría que haber algún tipo de programa al respecto, global de la universidad, que hiciera coordinarse todo ello" (E3). Además, se colabora con asociaciones y ONG externas: "Como la ONCE, Telefónica, Vodafone... la asociación de TDAH, con Frater Castellón que son físicos..." (E2).

Respecto a la orientación académica ofrecen: "Un acompañamiento de los estudiantes que han tenido mal rendimiento e informan al profesorado según la problemática y la necesidad que tengan de apoyo en el examen (...) derivación hacia asistencia psicológica, si procede (...) ayuda a nivel de organización de tiempos y técnicas de estudios" (E2).

En cuanto a la accesibilidad, la web y la accesibilidad física están garantizadas por ser una universidad de nueva creación. Los recursos específicos se consiguen mediante una fundación externa, Universia: "Hemos comprado ordenadores y dos emisoras FM porque tenemos dos estudiantes con problemática auditiva" (E2).

En la dimensión C (Prácticas), se considera fundamental mejorar la formación del profesorado sobre inclusión, pues: "Es algo transversal y obligatorio dentro de la confección de los planes de estudios" (E3).

Hay guías de accesibilidad y de contenidos transversales dentro de las diferentes titulaciones pero se trabaja más a nivel de departamento cuando surge la necesidad concreta: "Y decimos que es formación pero no es formación reconocida en el plan de formación y eso se queda en reuniones, pero eso es formación" (E2). Por ejemplo: "A principios de septiembre contactamos con el director o directora y le decimos: Tenéis un alumno con Asperger y se le explican las características... Pienso que hay que hacerlo de cada caso en petit comité o mediante una actuación más directa con esa profesora..." (E1).

Se considera necesario que el profesorado novel tenga una formación obligatoria: "Una introducción a qué son las personas con necesidades específicas o especiales... unos datos mínimos... es que hay 300 estudiantes y alguno os puede tocar. También deberían saber a quién recurrir y cómo" (E2). 


\subsection{Percepciones de los responsables de los servicios de la Fundación Universitaria Los Libertadores}

Sobre la dimensión A (Cultura), en este contexto existe una concienciación frente al enfoque del derecho a la educación, pero al definir educación inclusiva las opciones que dan los entrevistados tiene que ver directamente con discapacidad: "Es atender a las personas inválidas o con alguna enfermedad" (E4). Así, es evidente que el imaginario social sigue siendo de una persona con dificultades y desde la corporalidad, donde se "homogeniza a un grupo y se excluye a otros" (E8).

En el Proyecto Educativo Institucional Libertador (PEIL) se recoge el concepto de formación integral de los sujetos que integran la comunidad desde las dimensiones ética, moral, estética, social, política, física, artística, intelectual, cultural, religiosa, productiva con capacidades y potenciales como ser humano (PEIL, 2008). Así pues, relacionando los principios y la concepción de persona que se tiene a nivel institucional, se piensa en la equidad de oportunidades para garantizar el derecho a la educación en los diferentes programas de educación ofrecidos. Esto ha permitido que desde el 2013 se iniciara la atención educativa a poblaciones con discapacidad visual (2), auditiva (2) y Síndrome de Asperger (1) en los programas educativos de Licenciatura en Educación Especial y Diseño Gráfico.

El reconocimiento del concepto de inclusión está dado sólo al grupo de estudiantes que requieren adaptaciones para cumplir sus objetivos y que "los remiten los docentes al ver que no tienen las competencias para su espacio académico" (E6). Aunque apuntan que si se reconociera que "existen múltiples formas para aprender, como existe múltiples formas de ser, sería posible hablar de inclusión" (E8).

En cuanto a la dimensión B (Política), los entrevistados tienen claro que existe una legislación nacional que ampara el derecho a la educación y como entidad privada se ha tratado de garantizar que todos los estudiantes que acceden a la fundación reciban una atención educativa de calidad, por ello se establecen procesos para la permanencia. Sin embargo, hasta noviembre del 2016 no existía una política institucional: "Todos tenemos acciones que nos permiten garantizar que un estudiante pueda graduarse de manera oportuna superando las dificultades de aprendizaje o económicas y en muchos casos emocionales, pero no está institucionalizado como educación inclusiva o política de inclusión (E7)".

En el acceso se puede evidenciar que la matrícula no tiene restricción por condiciones de los aspirantes: "Sencillamente ingresan al programa a través de una entrevista y en ocasiones una prueba específica que no califica sino que sirve para orientar el proceso del nuevo estudiante" (E5).

Sin embargo, "no se cuenta con mecanismos para apoyar el ingreso de estudiantes sordos o con alguna condición" (E4), aunque "el sistema de alertas sí permite identificar al grupo de estudiantes que requiere un apoyo en psicología, psicopedagogía, trabajo social, y se ha avanzado en las remisiones gracias a los profesores consejeros de cada programa" (E4). Se está detectando una problemática relacionada con el aspecto social, emocional, económico o de aprendizaje, pues es una situación que aumenta cada semestre y, además, "cada vez los estudiantes llegan con mayor requerimiento en apoyos en lectura, escritura y hasta en la toma de decisiones" (E5).

En cuanto a la dimensión C (prácticas), se formulan interrogantes sobre si existe una sola forma de hacer el proceso de formación, pues en el momento de revisar los programas se preguntan "qué hacer con el que no puede memorizar, como realizar las clases si no me escucha y si es literal" (E8). Consideran que para buscar respuestas, lo esencial sería "conocer al estudiante" (E4), es decir, una de las mejores prácticas es cuando un profesor identifica las capacidades de sus estudiantes.

\subsection{Percepciones de los responsables de los servicios de apoyo de la Université de Reims}

Respecto de la dimensión A (Cultura), el término inclusión es desconocido por los informantes y afirman: "es una palabra que no entiendo mucho" (E11), pero la relacionan con "la acogida de los estudiantes con discapacidad para que puedan seguir su formación [así como] una posibilidad de dar igualdad de oportunidades a los estudiantes con discapacidad" (E11), y consideran que es más una acción individual, una actitud moral que los lleva a ayudar a los estudiantes que tienen dificultades específicas más que una política institucional. En el contexto francés, donde las universidades no practican la selección, los entrevistados se muestran sensibles a la noción de igualdad de oportunidades (término más utilizado durante las entrevistas) en lugar del de "valoración de la diversidad". 
Sobre la dimensión B (Política) consideran que las cuestiones organizativas se encauzan a través del Servicio a la Discapacidad (SAD), que "funcionan en red" (E10) en el marco de una asociación nacional, la cual desarrolla (con los diferentes ministerios implicados) las investigaciones y la información. El Servicio a la discapacidad de la URCA se ocupa de 177 estudiantes. Más de la mitad (52\%) tiene una pensión y a más de tres cuartos (77.9\%) se les realizan adaptaciones en los exámenes (disponen de más tiempo que el resto).

En los últimos años el número de estudiantes con discapacidad ha ido en aumento: "En 2012 había cuatro o cinco estudiantes por facultad y ahora son de doce a quince cada año" (E13). Los estudiantes con discapacidad ya reciben información del servicio de la discapacidad desde el instituto. Por tanto, ya están en contacto cuando se matriculan y entonces se realiza un plan personalizado a partir del examen de su situación por una comisión compuesta de personal médico, social y profesorado. El servicio avisa a los responsables de cada facultad, aunque no siempre es ese el procedimiento, pues, en algunos casos, los estudiantes son descubiertos por el profesorado. En cuanto a la cultura colaborativa, existe colaboración entre el profesorado y el SAD informando a las facultades de los estudiantes matriculados si hay que proporcionar recursos adaptados como en el caso siguiente: "Teníamos un problema porque los primeros años se mira mucho por el microscopio. El servicio adquirió una cámara para conectarla al microscopio, de manera que el estudiante puede mirar sin fatigarse" (E13).

No se alude a la colaboración entre el profesorado de estudiantes con discapacidad, aunque se mencionaron varios ejemplos de colaboración entre estudiantes. Por ejemplo, uno de los responsables dice: "Este año, por primera vez, los estudiantes se han puesto en contacto conmigo diciendo: estamos en un buen grupo que funciona bien y eso que hay una estudiante con dificultades y nos turnamos con ella en función de las clases, porque algunas son difíciles..." (E11). En cuanto a la accesibilidad, tal y como exige la ley, se han hecho esfuerzos para garantizar la accesibilidad física de los locales antiguos o recientes, aunque todavía hay problemas, como "fuertes pendientes en las rampas o retrasos en la reparación de los ascensores" (E9).

En cuanto a la dimensión C (Prácticas) afirman que no es frecuente que el profesorado modifique sus prácticas docentes y lo justifican en la igualdad de trato a todos los estudiantes, lo que representa una dificultad para que algunos estudiantes gravemente afectados sigan con sus estudios. No se adapta el contenido de los exámenes, pero sí son frecuentes algunas adaptaciones, como: "Tiene un tiempo extra, es decir, de dos horas tienen 40 minutos más" (E13). En cuanto a la formación del profesorado, no es obligatoria y a la pregunta de si es necesaria una formación para la acogida de estudiantes con discapacidad, responden con evasivas: “Por qué no sobre lo ideal?" (E12); "estaría bien conocer cómo reaccionar ante los estudiantes con discapacidad" (E13) pero insisten en que es una actitud personal y no ven la necesidad de una formación pedagógica particular.

\section{Discusión y conclusiones}

Los servicios de apoyo tienen un importante rol para que las instituciones desarrollen una cultura inclusiva. Pero para ello es fundamental que asuman la conceptualización de la inclusión. En este sentido, el personal de los servicios de apoyo se sitúa en tres posiciones muy diferentes. En la Universitat Jaume I parece que la definición teórica está más clara aludiendo a las particularidades de las personas, al derecho a la igualdad de oportunidades y a la necesidad de articular los recursos y la respuesta educativa con relación a las prácticas docentes, a pesar de que el desarrollo de propuestas prácticas está muy ligado al modelo de necesidades educativas especiales. En el contexto de Los Libertadores y de la Université de Reims, la conceptualización está asociada a las personas con discapacidad, como personas con dificultades, más propio del modelo integrador (Thomazet, 2015). Estos resultados nos llevan al primer desafío: la claridad de la definición en relación con la idea de inclusión, lo cual supone un reto de por sí de carácter internacional apoyando la idea de acoger con satisfacción la diversidad de todos los estudiantes. El objeto es eliminar la exclusión social, consecuencia de las actitudes y respuestas a la diversidad de raza, clase social, origen étnico, religión, género y capacidades, desde un concepto amplio de la educación inclusiva (Armstrong, Armstrong y Barton 2000; Prets y Weber, 2005).

Este planteamiento nos lleva al segundo desafío de las universidades: ¿Cómo se concreta el valor de la inclusión en las políticas, estrategias, procesos y programas? En ese sentido, los resultados apuntan a una 
mayor concreción normativa en las políticas de la Fundación Los Libertadores, que explicita un objetivo de universidad inclusiva en su proyecto educativo, lo cual va a permitir un mayor progreso en los desarrollos prácticos del proceso. No sucede lo mismo en las otras dos universidades, en las que la inclusión no se formula de manera explícita sino que las normativas, planes y programas se enfocan a garantizar la igualdad de oportunidades y atender a los estudiantes "con necesidades".

En los tres casos se alude a cómo los servicios de apoyo facilitan la inclusión en la universidad mediante la oferta de recursos específicos, la atención directa al estudiantado, la sensibilización a la comunidad universitaria, un sistema (programa) de acceso centralizado que permite la detección y el apoyo a los estudiantes, gran coordinación con recursos y entidades externas de carácter educativo y social. Sin embargo, en los tres casos se echa en falta más coordinación entre los servicios y programas internos de las propias universidades. Ello nos lleva a plantear el tercer desafío: establecer mecanismos de coordinación entre diferentes servicios internos que tienen por objeto la inclusión y acogida del alumnado, en particular el más vulnerable.

Por otra parte, los resultados también muestran algunas barreras intangibles, como las actitudinales y las pedagógicas. Entre las primeras, se nombra la falta de cooperación de los docentes para realizar las adecuaciones necesarias, con lo cual el cuarto desafío estaría en relación con el cambio actitudinal hacia las formas de enseñar de los docentes universitarios que, según Bausela (2002), es lo más difícil de cambiar.

Ello está directamente relacionado con las barreras que experimenta el profesorado en relación con su falta de formación pedagógica. Este es uno de los puntos de mayor discrepancia del estudio, pues mientras unos se inclinan por una formación obligatoria del profesorado novel sobre aspectos generales relacionados con la discapacidad, otros reclaman un acompañamiento "a la carta" para los casos específicos a partir de las singularidades del estudiantado, e incluso algunos no la consideran necesaria. Sobre este aspecto tampoco encontramos acuerdo en la literatura, pues mientras algunos autores proponen una formación más centrada en el aprendizaje sobre las discapacidades (Barazandeh, 2005; Kraska, 2003), otros proponen cursos para incorporar a la enseñanza universitaria los planteamientos del DUA (Alba, Zubillaga y Sánchez, 2015), así como el desarrollo de grupos de innovación docente en los que se integra el alumnado para discutir sobre sus necesidades (Sarkar, 2016). En definitiva, el quinto desafío tiene que ver con el desarrollo de las competencias pedagógicas del profesorado, como una oportunidad para innovar en estrategias docentes que posibiliten a los profesionales de la educación superior fortalecer su práctica desde una mirada pedagógica inclusiva.

\section{Referencias}

Alba, C., Zubillaga, A. y Sánchez, J. M. (2015). Tecnologías y Diseño Universal para el Aprendizaje (DUA): experiencias en el contexto universitario e implicaciones en la formación del profesorado. Revista Latinoamericana de Tecnología Educativa, 14(1) 89-100. doi:10.17398/1695-288X.14.1.89

Alonso, A. y Díez, E. (2008). Universidad y discapacidad: indicadores de buenas prácticas y estándares de actuación para programas y servicios. Siglo Cero. Revista Española sobre Discapacidad Intelectual, 39(2), 8298. Recuperado de http://sid.usal.es/10763/8-2-6

Armstrong, F., Armstrong, D. y Barton, L. (2000). Inclusive education: policy, contexts, and comparative perspectives. Londres: Fulton.

Barazandeh, G. (2005). Attitudes toward disabilities and reasonable accommodation at the university. The Undergraduate Research Journal, 7, 1-12.

Bausela, E. (2002). Atención a la diversidad en la educación superior. Revista de Curriculum y Formación del Profesorado, 6(1), 1-11. Recuperado de https://recyt.fecyt.es/index.php/profesorado/article/view/41971/23954

Booth, T. y Ainscow. M. (2002). Guía para la evaluación y mejora de la educación inclusiva: desarrollando el aprendizaje y la participación en las escuelas (A. L. López, D. Durán, G. Echeita, C. Giné, E. Miquel, S. Moratalla y M. Sandoval, Trads.). Madrid: CSIE y Consorcio Universitario para la Educación Inclusiva. 
Borland, J. y James, S. (1999). The learning experience of students with disabilities in higher education. Disability y Society, 14(1), 85-101. doi:10.1080/09687599926398

Castellana, M. y Sala, I. (2005). La universidad ante la diversidad en el aula. Aula Abierta, 32(85), 57-84. Recuperado de https://www.unioviedo.es/reunido/index.php/AA/issue/viewlssue/1033/131

Douchet, A., Aubree, L. y Mabaka, P. (2013). L'inclusion des étudiants en situation de handicap dans les universités à l'échelle mondiale [La inclusión de estudiantes con discapacidad en universidades del mundo]. Lille: Université Catholique de Lille.

Fundación Universitaria Los Libertadores. (2018) Proyecto Educativo Institucional Libertador (PEIL). Recuperado de https://www.ulibertadores.edu.co/images/documentos-institucionales/documentos/ proyecto-educativo-institucional-2018-2.pdf

Grosstephan, V. y Philippot, T. (2015). L'inclusion des étudiants en situation de handicap à l'université en France: d'une demande sociale aux pratiques des acteurs, une étude de cas. En S. Guido (Comp.), ¿Integración-inclusión. Cuáles intervenciones educativas? Memorias del Primer Coloquio Internacional Integración-inclusión, Bogotá, 2014.

Inspection Générale de l'Administration de l'Éducation Nationale et de la Recherche (2006). La politique d'accueil des étudiants handicapés (Rapport 2006-50) [La política de acogida para estudiantes con discapacidad (Informe 2006-50). París: Ministerio de Educación Nacional.

Kraska, M. (2003). Postsecondary students with disabilities and perception of faculty Members. The Journal for Vocational Special Needs Education, 25(2), 11-19.

Leyser,Y., Greenberger, L., Sharoni, V. y Vogel, G. (2011). Students with disabilities in teacher education: changes in faculty attitudes toward accommodations over ten years. International Journal of Special Education 26(1), 162-174. doi:10.5539/ies.v6n12p74

Moriña, A., Cortés-Vega, M. D. y Molina, V. (2015). Faculty training: an unavoidable equirement for approaching more inclusive university classrooms. Teaching in Higher Education, 20(8), 795-806. doi:10.1080/13562517.2015.1085855

Moswela, E. y Mukhopadhyay, S. (2011). Asking for too much? The voices of students with disabilities in Botswana. Disability y Society, 26(3), 307-319. doi:10.1080/09687599.2011.560414

Prets, C. y Weber, H. (2005). Intégration et handicaps: la situation européenne [Integración y desventajas: la situación europea]. Reliance, 16, 54-60. Recuperado de https://www.cairn.info/revue-reliance-2005-2page-54.htm

Prowse, S. (2009). Institutional construction of disabled students. Journal of Higher Education Policy and Management, 31(1), 89-96. doi:10.1080/13600800802559302

Riddell, S. y Weedon, E. (2014). Disabled students in higher education: discourses of disability and the negotiation of identity. International Journal of Educational Research, 63, 38-46.

doi:10.1016/j.ijer.2013.02.008

Rodríguez, L. D. (2015). La inclusión educativa de personas con discapacidad: políticas y prácticas en la educación superior. Estudio comparativo entre la Universidad Católica de Córdoba-Argentina y la Fundación Universitaria Los Libertadores-Colombia. Observatorio Pedagógico de la Infancia, (15), 9-15.

Sabourin, P. (2009). L'analyse de contenu. En B. Gauthier (Ed.), Recherche sociale: de la problématique à la collecte des données (pp. 415-444) [Investigación social: de la problemática a la recolección de datos]. Québec: Universidad de Québec.

Sarkar, R. (2016). Inclusive university: a way out to ensure quality, equity, and accessibility for students with disabilities in higher education. International Journal of Advanced Research, 4(4), 406-412.

doi:10.21474/IJAR01/312

Shevlin, M., Kenny, M. y Mcneela, E. (2004). Participation in higher education for students with disabilities: an irish perspective. Disability \& Society, 19(1), 15-30. doi:10.1080/0968759032000155604 
Thomazet, S. (2015). L'intégration a des limites. Pas l'école inclusive. En S. Guido (Comp.), ¿Integracióninclusión. Cuáles intervenciones educativas? Memorias del Primer Coloquio Internacional Integracióninclusión, Bogotá, 2014.

Yarza, A., Rojas, H. y López, L. (2014). La discapacidad y Educación Superior. Las contiendas por la participación y la ciudadanía en las reformas neoliberales de la universidad y la Educación Superior en Colombia. Revista Latinoamericana De Educación Inclusiva, 8(1), 35-49. 